\title{
Secondary Metabolites from Escovopsis weberi and Their Role in Attacking the Garden Fungus of Leaf-Cutting Ants
}

\author{
Basanta Dhodary, ${ }^{[a]}$ Michele Schilg, ${ }^{[b]}$ Rainer Wirth, ${ }^{[b]}$ and Dieter Spiteller*[a]
}

\begin{abstract}
The specialized, fungal pathogen Escovopsis weberi threatens the mutualistic symbiosis between leaf-cutting ants and their garden fungus (Leucoagaricus gongylophorus). Because $E$. weberi can overwhelm L. gongylophorus without direct contact, it was suspected to secrete toxins. Using NMR and mass spectrometry, we identified several secondary metabolites produced by E. weberi. E. weberi produces five shearinine-type indole triterpenoids including two novel derivatives, shearinine $L$ and shearinine $M$, as well as the polyketides, emodin and cycloarthropsone. Cycloarthropsone
\end{abstract}

\section{Introduction}

Attine ants (subfamily Myrmicinae, tribe Attini) evolved the practice to cultivate the fungus Leucoagaricus gongylophorus (Agaricales: Leucocoprineae). Among attine ants, the genera Acromyrmex and Atta are unique because they provide their fungal symbiont with fresh plant material as growth substrate. In turn the fungus serves the ants as primary food source. ${ }^{[1]}$

Microbial pathogens from the surrounding soil of the nest and microorganisms brought into the nest because of the foraging behavior ${ }^{[2]}$ of the leaf-cutting ants potentially threaten the garden fungus as well as the leaf-cutting ants' survival. ${ }^{[3]}$ Therefore, leaf-cutting ants control the growth of adverse microbes in their fungus gardens by chemical treatment ${ }^{[4]}$ and weeding behavior. ${ }^{[5]}$ In addition antibiotic-producing Actinomyces symbionts support leaf-cutting ants in the defense against noxious invaders. ${ }^{[6]}$ Even so, a broad variety of detrimental bacteria, filamentous fungi and yeasts occur in the nest of leaf-cutting ants. ${ }^{[2,7]}$ In particular, the specialized fungal parasite Escovopsis (Ascomycota, Pezizomycotina: anamorphic Hypocreales) attacks the fungus garden of leaf-cutting ants. ${ }^{[7 b]}$ Escovopsis fungi have never been isolated outside of the fungus-growing ants' ecosystem and have been established to have coevolved

[a] B. Dhodary, Prof. Dr. D. Spiteller

Chemical Ecology/Biological Chemistry, University of Konstanz

Universitätsstraße 10, 78457 Konstanz (Germany)

E-mail:dieter.spiteller@uni-konstanz.de

[b] M. Schilg, Dr. R. Wirth

Plant Ecology and Systematics, Technical University Kaiserslautern

Erwin-Schrödingerstraße 13, 67653 Kaiserslautern (Germany) and emodin strongly inhibited the growth of the garden fungus $L$. gongylophorous at 0.8 and $0.7 \mu \mathrm{mol}$, respectively. Emodin was also active against Streptomyces microbial symbionts $(0.3 \mu \mathrm{mol})$ of leaf-cutting ants. Shearinine $L$ instead did not affect the growth of $L$. gongylophorus in agar diffusion assays. However, in dual choice behavioral assays Acromyrmex octospinosus ants clearly avoided substrate treated with shearinine $L$ for the garden fungus after a $2 \mathrm{~d}$ learning period, indicating that the ants quickly learn to avoid shearinine $\mathrm{L}$.

in association with fungus-growing ants and their cultivated fungi. ${ }^{[6 a]}$ In comparison to their closest known relatives, Escovopsis adapted to the mycoparasitic lifestyle, for example, by loss of genes coding for plant-material-degrading enzymes. ${ }^{[8]}$ Moreover, different Escovopsis strains can destroy the fungus gardens of Atta and Acromyrmex leaf-cutting ants. ${ }^{\left[{ }^{[9}\right.}$

Reynolds and Currie established in 2004 that Escovopsis weberi does not compete for the plant material brought into the nest by the leaf-cutting ants to feed $L$. gongylophorus but it directly consumes the mutualistic fungus. ${ }^{[10]}$ E. weberi degrades the hyphae of L. gongylophorus before direct physical interaction, suggesting that the pathogen secretes toxins and/ or enzymes that can break down host mycelium before contact occurs. ${ }^{[10]}$ The recently sequenced $E$. weberi genome encodes for a variety of secondary metabolite biosynthesis gene clusters. ${ }^{[8]}$ Some of these gene clusters including polyketide synthase gene clusters were significantly up-regulated when growing with L. gongylophorus. ${ }^{[8]}$ Moreover, crude extracts of several Escovopsis species were reported to inhibit the growth of L. gongylophorus. ${ }^{[11]}$ In a co-cultivation study of $E$. weberi with microbial symbionts of leaf-cutting ants Férnandez-Marín et al. discovered that $E$. weberi produces several shearinines. ${ }^{[12]}$

However, until now, no other secondary metabolites from E. weberi have been identified and it has not been established which compounds from $E$. weberi contribute to overpower the mutualistic fungus of leaf-cutting ants. ${ }^{[11,12]}$ Here, we present the isolation and identification of secondary metabolites from $E$. weberi and evaluate their biological function, in particular their impact on L. gongylophorus and A. octospinosus leaf-cutting ants. 


\section{Results}

\section{Identification of secondary metabolites from $E$. weberi}

Methanol extracts of $E$. weberi grown on SFM agar plates at $28^{\circ} \mathrm{C}$ for $14 \mathrm{~d}$ were analyzed by high performance liquid chromatography (HPLC) coupled to a diode array detector and an electrospray mass spectrometer (LC-DAD-ESI-MS). The base peak chromatogram and the UV chromatogram (254 nm) (Figures $1 \mathrm{~A}$ and $\mathrm{S} 1$ in the Supporting Information) revealed a

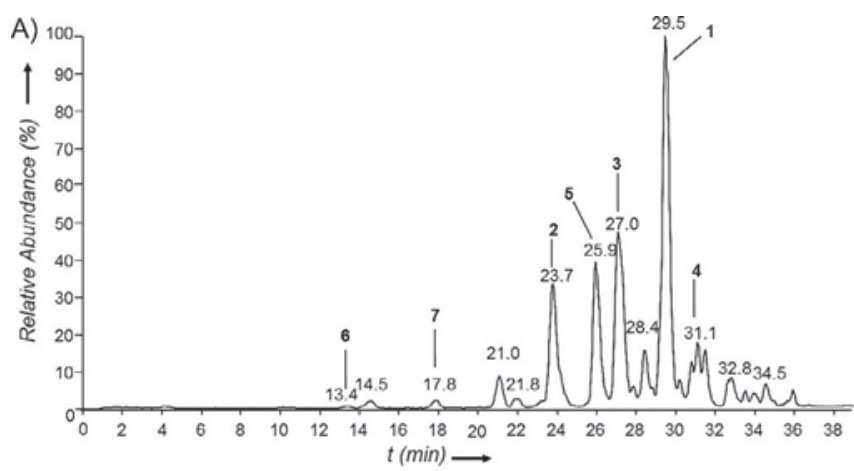

B)
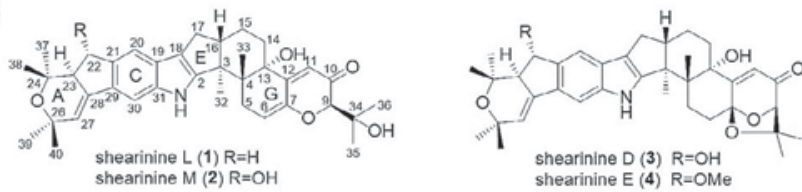
shearinine $E$ (4) $R=O M$ shearinine $A(5) \mathrm{R}=\mathrm{H}$
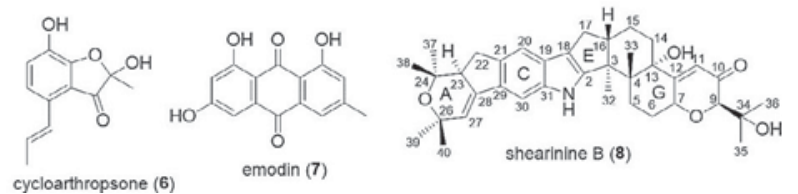

Figure 1. A) LC-negative-ESI-MS base peak chromatogram of a methanol extract of $E$. weberi. Labelled peaks were identified as shearinine $L(1)$, shearinine $M(2)$, shearinine $D(3)$, shearinine $E(4)$, shearinine $A(5)$, cycloarthropsone (6), and emodin (7). B) Structures of the identified secondary metabolites from $E$. weberi and shearinine B (8) for comparison.

series of intense peaks. The compounds causing the most prominent peaks 1-7 were purified by silica gel column chromatography (CC), Sephadex LH-20 CC and reversed phase HPLC coupled with a fraction collector in order to elucidate their structures by mass spectrometry and NMR. The purified secondary metabolites comprised five shearinines including two novel shearinines ( 1 and 2 ), cycloarthropsone (6), and emodin (7) (Figure 1B). E. weberi also produces these secondary metabolites when it grows together with $L$. leucoagaricus on PDA medium mimicking the attack of the garden fungus by E. weberi (Figure S31 B).

\section{Shearinines}

Several shearinines have been recently identified as secondary metabolites from $E$. weberi. ${ }^{[12]}$ However, we observed additional prominent peaks $\mathbf{1}$ and $\mathbf{2}$ in the LC-DAD-ESI-MS profile that turned out to be novel shearinine derivatives.

Shearinines were detectable in SFM agar plates inoculated with $E$. weberi after $3 \mathrm{~d}$ at $28^{\circ} \mathrm{C}$. The amount of shearinines increased significantly with incubation time, reaching a maximum concentration of approximately $8 \mu \mathrm{g} \mathrm{mL}^{-1}$ SFM medium of 1 after day 9 . Shearinine L (1) was obtained as a white amorphous powder. Its molecular formula was established to be $\mathrm{C}_{37} \mathrm{H}_{45} \mathrm{NO}_{5}$ by HR-ESI-MS corresponding to 16 double bond equivalents (DBE).

The ${ }^{1} \mathrm{H}$ NMR spectrum (Figure 2, Figure S3) showed the presence of signals for eight aliphatic methyl singlets which are characteristic for a shearinine-type indole triterpenoid as well as two indicative aromatic proton singlets at $\delta_{\mathrm{H}}=7.16(\mathrm{H}-20)$ and $7.35(\mathrm{H}-30)$. The ${ }^{13} \mathrm{C}$ NMR spectrum (Figure $\mathrm{S} 4$ ) revealed 37 carbon resonances corresponding to eight methyl groups, five methylene groups, and eight methine groups. Furthermore, among the 16 quaternary carbons, the signal at $\delta_{C}=197.6$ (C10) indicated the presence of an unsaturated keto group. The ${ }^{1} \mathrm{H}$ and ${ }^{13} \mathrm{C}$ NMR data together with $\mathrm{HMBC}$ correlation analysis,
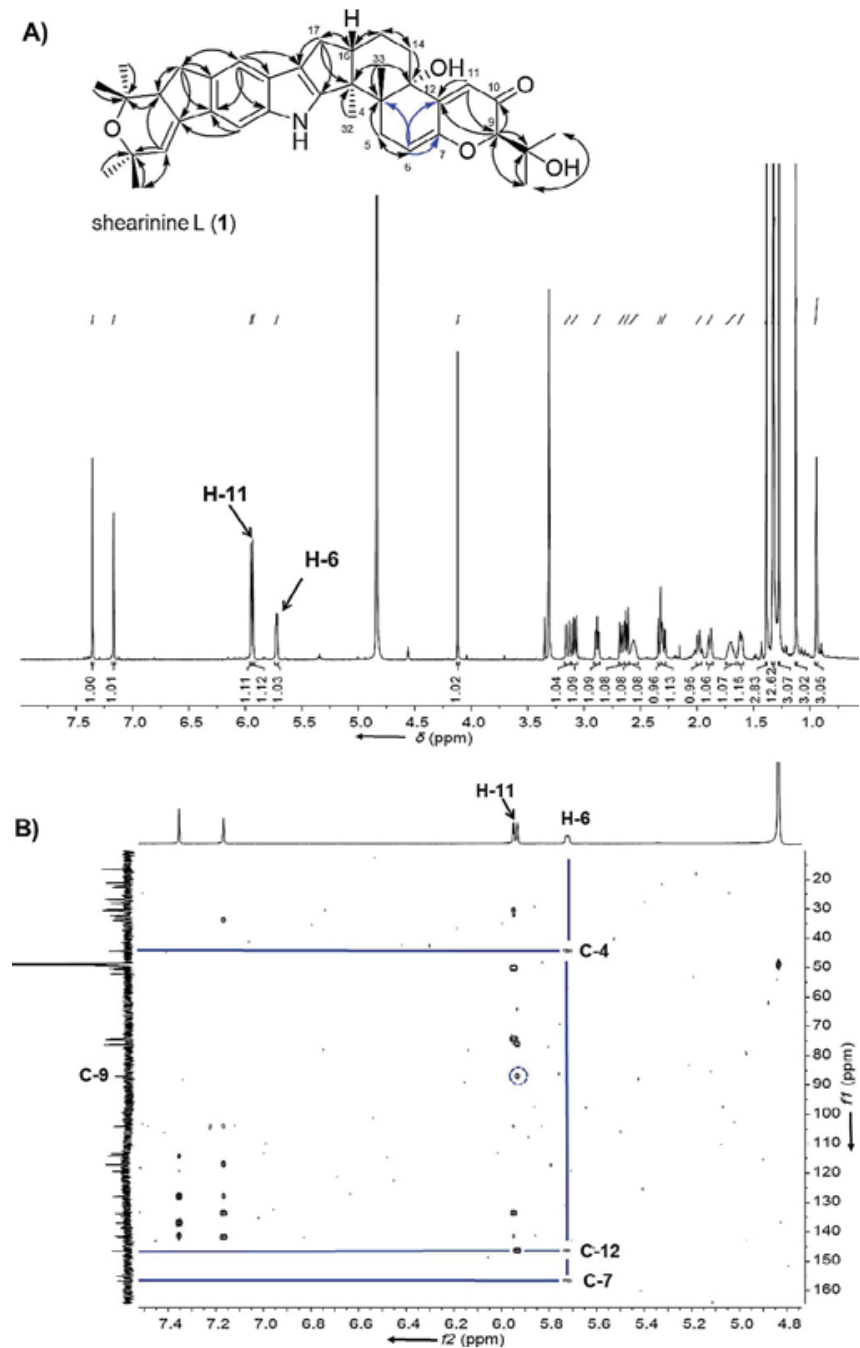

Figure 2. A) ${ }^{1} \mathrm{H}$ NMR of shearinine $\mathrm{L}(1)$, B) key ${ }^{1} \mathrm{H}-{ }^{1} \mathrm{H}$ COSY and ${ }^{1} \mathrm{H}-{ }^{13} \mathrm{C} H M B C$ correlations of shearinine $L(\mathbf{1})$. 
suggested that the structure of $\mathbf{1}$ is closely related to that of shearinine $B(8) .{ }^{[13]}$ Compound 1 and shearinine $B(8)^{[13]}$ differ only in the ring $G$ (Figure 2). Compound 1 bears a double bound between $\mathrm{C}-6$ and $\mathrm{C}-7$ that causes the appearance of one characteristic olefinic proton at $\delta_{\mathrm{H}}=5.72(\mathrm{H}-6)$. The HMBC correlations between $\mathrm{H}-6$ and $\mathrm{C}-4\left(\delta_{\mathrm{C}}=44.3\right), \mathrm{C}-7 \quad\left(\delta_{\mathrm{C}}=156.8\right)$ and $\mathrm{C}-12\left(\delta_{\mathrm{C}}=146.5\right)$ (Figure $2 \mathrm{~B}$, Figure S7) further confirmed the position of the double bond. Thus, 1 was determined as $\Delta 6,7$-shearinine $B(8)$ and called shearinine $L$. The relative configuration of shearinine $L(1)$ was clarified by NOESY spectrum analysis (Figure S8). The presence of NOE correlations between $\mathrm{H}_{3}-32$ to $\mathrm{H}-17 \alpha$ and also between $\mathrm{H}-16$ to $\mathrm{H}_{3}-33$ indicated a trans-3,16-ring fusion.

NOE correlations between $\mathrm{H}_{3}-33$ and $\mathrm{H}-14 \beta$ as well as between $\mathrm{H}-11$ and $\mathrm{H}-14 \alpha$ suggested the $\alpha$-orientation of the hydroxy group at $\mathrm{C}-13$ (Figure S9). Based on the study by $\mathrm{Xu}$ et al. ${ }^{[14]}$ and biosynthetic considerations we suggest the absolute configuration of shearinine $L(1)$ to be identical to that of shearinine $\mathrm{D}(3)$ which is also produced by $E$. weberi.

The HR-ESI-MS of compound 2 confirmed its molecular formula to be $\mathrm{C}_{37} \mathrm{H}_{45} \mathrm{NO}_{6}$ (16 DBE). Thus, 2 contains one oxygen atom (16 amu) more compared to shearinine $L(1)$. The ${ }^{1} \mathrm{H}$ and ${ }^{13} \mathrm{C}$ NMR signals of 2 (Figure S11 and S12) were similar with those of shearinine $L(1)$ except for the presence of a single broad doublet signal at $\delta_{\mathrm{H}}=4.90(1 \mathrm{H}, J=5.7 \mathrm{~Hz}, \mathrm{H}-22)$ instead of the two signals $\delta_{\mathrm{H}}=3.09(1 \mathrm{H}, J=15.3,9.2 \mathrm{~Hz}, \mathrm{H}-22), 2.60-$ $2.64(1 \mathrm{H}, \mathrm{m}, \mathrm{H}-22)$ in shearinine $\mathrm{L}$ (1) (Figure S3 and S11). This suggested that compared to shearinine $L(1)$, compound 2 contains a hydroxylated methine moiety at carbon C-22. The position of the hydroxy group at C-22 was further confirmed by HMBC correlations between $\mathrm{H}-22$ and $\mathrm{C}-23\left(\delta_{\mathrm{C}}=60.3\right), \mathrm{C}-24$ $\left(\delta_{\mathrm{C}}=75.6\right), \mathrm{C}-20\left(\delta_{\mathrm{C}}=114.2\right), \mathrm{C}-21 \quad\left(\delta_{\mathrm{C}}=132.0\right), \mathrm{C}-28\left(\delta_{\mathrm{C}}=137.3\right)$ and C-29 $\left(\delta_{C}=139.2\right)$ (Figure S15). Thus, the structure of 2 was determined as 22-hydroxy-shearinine L. We named the new compound shearinine $M$. The NOESY spectrum exhibited correlations between $\mathrm{H}-22$ and $\mathrm{H}_{3}-38$ and between $\mathrm{H}-23$ and $\mathrm{H}_{3}-37$ (Figure S16), which implies that $\mathrm{H}-22$ and $\mathrm{H}-23$ are on opposite faces of ring $\mathrm{B}$. The ${ }^{1} \mathrm{H}$ and ${ }^{13} \mathrm{C}$ NMR data at $\mathrm{C}-22$ and $\mathrm{C}-23$ of 2 were also in good agreement with those of shearinine $D_{1}{ }^{[15]}$ indicating the relative $\mathrm{H}-22 \beta$ and $\mathrm{H}-23 \alpha$ configurations. The relative configuration of ring fusions in shearinine $M(2)$ is identical to shearinine $L(1)$ based on the NOESY correlations: $\mathrm{H}_{3}-32 / \mathrm{H}$ $17 \alpha, \mathrm{H}-16 / \mathrm{H}_{3}-33, \mathrm{H}_{3}-33 / \mathrm{H}-14 \beta$ and $\mathrm{H}-11$ and $\mathrm{H}-14 \alpha$ (Figure $\mathrm{S17B}$ ). Because shearinine $\mathrm{M}(2)$ is closely related to shearinine $L(1)$ and $D(3)$ it is expected to have the same absolute configuration as shearinine $\mathrm{D}(\mathbf{3}) .^{[14]}$

Compounds 3-5 were identified as shearinine-type indole triterpenoids, namely shearinine $D(3)$, shearinine $E(4)$, and shearinine A (5) (SI), which have been characterized before from Penicillium species or its teleomorph Eupenicillium species. $^{[13-15]}$

\section{Cycloarthropsone (6)}

$3.2 \mathrm{mg}$ of compound 6 (ca. $6 \mu \mathrm{g} \mathrm{mL}^{-1}$ SFM medium) were isolated. 6 was an aromatic compound with the molecular formula $\mathrm{C}_{12} \mathrm{H}_{12} \mathrm{O}_{4}$ and seven DBE. Its ${ }^{1} \mathrm{H}$ NMR spectrum exhibited two ortho-coupled aromatic protons, signals corresponding to an (E)-propenyl chain and a methyl singlet (Figure S23). The NMR data and MS/MS fragmentation (Figure S25) indicating loss of $\mathrm{H}_{2} \mathrm{O}$ and $\mathrm{CO}$ suggested 6 to be a benzofuranone derivative. Moreover, GC-MS analysis after derivatization with $\mathrm{N}$-methyl- $\mathrm{N}$ (trimethylsilyl)trifluoroacetamide (MSTFA) (Figure S26) exhibited a molecular ion $\left(\mathrm{M}^{+}\right)$at 364 , which pointed to the presence of two hydroxy groups. Thus, compound 6 was identified as cycloarthropsone that had been identified previously from the fungus Arthropsis truncata. The reported NMR data matched perfectly with ours. ${ }^{[16]}$

\section{Emodin (7)}

Compound 7 was obtained as orange needles (ca. $6 \mu \mathrm{g} \mathrm{mL}^{-1}$ SFM medium). For the aromatic compound the molecular formula $\mathrm{C}_{15} \mathrm{H}_{10} \mathrm{O}_{5}$ and 11 DBE were deduced. The UV spectrum of 7 in methanol (Figure S30) revealed four absorption maxima at 216, 256, 308 and $461 \mathrm{~nm}$ that are characteristic for anthraquinones. The ${ }^{1} \mathrm{H}$ NMR spectrum exhibited four meta-coupled aromatic proton signals and a methyl singlet signal (Figure S28). Further analysis of the ${ }^{13} \mathrm{C}$ NMR data (Figure S29) and comparison of the retention time with an authentic reference confirmed the compound to be emodin (7).

\section{Comparison of secondary metabolite profiles from $E$. weberi with Escovopsis aspergilloides}

E. aspergilloides that has also been identified from nests of attine ants ${ }^{[17]}$ is closely related to $E$. weberi. This parasitic fungus morphologically differs from $E$. weberi by its globose shaped, rather than clavate shaped phialide-vesicles. ${ }^{[18]}$ However, the metabolite profiles of methanol extracts of both Escovopsis strains turned out to be very similar (Figure S31 A). Both strains produced shearinines (1-5), cycloarthropsone (6), and emodin (7). The amount of the compounds in both Escovopsis strains may slightly vary.

\section{Antimicrobial activity of secondary metabolites from $E$. weberi}

In order to evaluate the function of the identified secondary metabolites, cycloarthropsone (6), emodin (7), and shearinine $L(1)$ were tested in agar diffusion assays against selected organisms present in the leaf-cutting ants' nests. In particular, the potential to overwhelm L. gongylophorus was addressed.

Cycloarthropsone (6) and emodin (7) inhibited the growth of L. gongylophorus, causing 14 and $10 \mathrm{~mm}$ inhibition zones, at 0.82 and $0.66 \mu \mathrm{mol}$, respectively (Figure 3, Figure S35-36). However, shearinine L (1) was not active at $0.31 \mu \mathrm{mol}$ (Figure S37). $0.30 \mu \mathrm{mol}$ emodin (7) inhibited the Streptomyces symbionts of leaf-cutting ants Streptomyces sp. 28_1 and Streptomyces sp. 26_1 causing inhibition zones of $10 \mathrm{~mm}$ and $7 \mathrm{~mm}$, respectively (Figure S32-S33). Moreover, none of the compounds tested showed activity against Pseudonocardia sp. Ao19, Fusarium equiseti, and Phialophora fastigiata. ${ }^{[19]}$ 


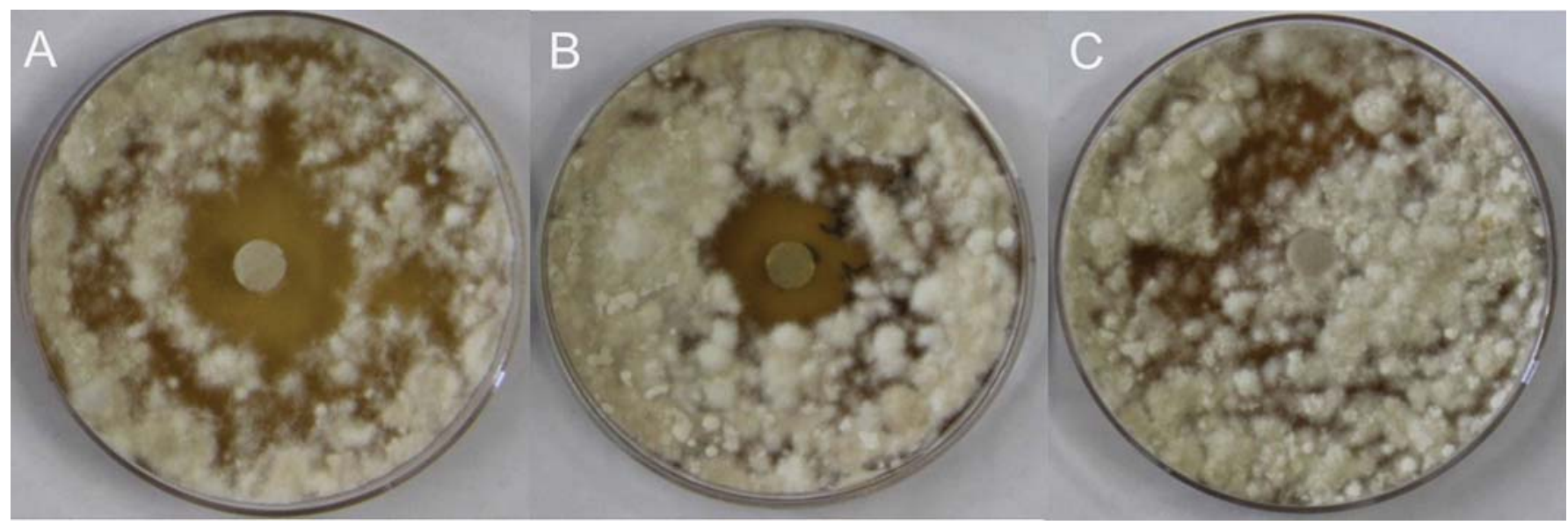

Figure 3. Agar diffusion assays against L. gongylophorous, on PDA agar plates (5.5 cm diameter) after $18 \mathrm{~d}$ of growth: A) $0.82 \mu \mathrm{mol}$ cycloarthropsone (6) on paper disk; B) $0.66 \mu \mathrm{mol}$ emodin (7) on paper disk, and C) control.

Response of $A$. octospinosus ants to shearinine $L(2)$

A. octospinosus ants from subcolonies were offered oat flakes as a substrate for their garden fungus. These flakes were either impregnated with shearinine $L$ (1) $(10 \mu \mathrm{g} /$ flake) or control flakes in a dual-choice assay. ${ }^{[20]}$ The percentage of flakes removed by the ants from the treated and the untreated pile of oat flakes, respectively, was determined (removal rate). The oat flakes were transported by $A$. octospinosus workers into the subcolonies and processed into small pieces that were incorporated across the garden to cultivate $L$. gongylophorus. Oat flake choice by $A$. octospinosus workers was strongly influenced by the shearinine L (1) treatment [Figure 4, GLMM (general linear mixed models) choice assay effect of treatment: $F_{1,18}=19.59$; $p<0.001]$. Notably, preference for the oat flakes reversed during the course of the assay from treated to untreated oat

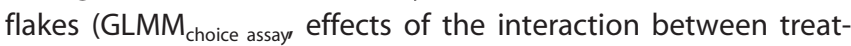
ment and day: $F_{4,18}=20.93 ; p<0.001$ ), although day per se had no significant effect due to low replication (GLMM choice assay;

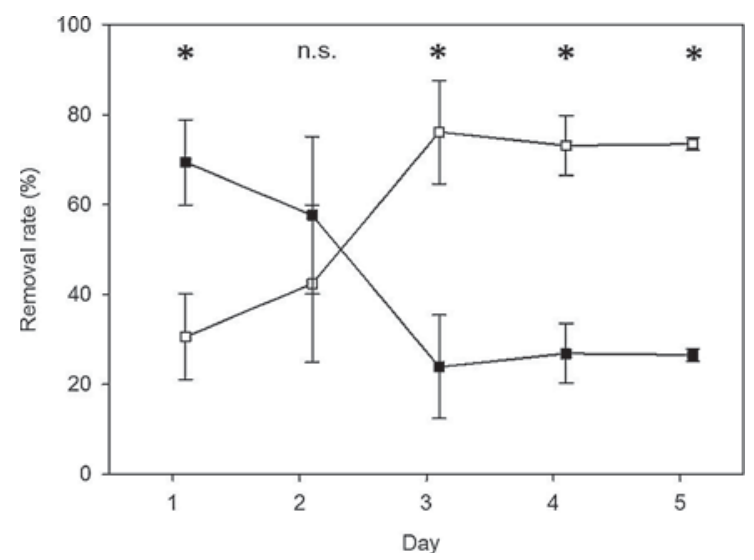

Figure 4. Dual choice assays with $A$. octospinosus leaf-cutting ants. The removal rate (percentage of oat flakes removed by the ants) of shearinine $L$ treated oat flakes versus untreated flakes is shown. Black squares (shearinine $L$ treated oat flakes) and white squares (untreated oat flakes) represent the mean values, error bars indicate the standard deviation based on 3 replicates. ${ }^{*}=$ statistically significant, n.s. $=$ not significant. effect of day: $\left.F_{4,18}=0.00 ; p>0.05\right)$. On day one, the ants significantly preferred treated over control flakes (ca. $70 \%$ versus $30 \%)$. Reversely, from day 3 onwards, shearinine $L$ treated oat flakes were strongly rejected (ca. $75 \%$ on days 3,4 , and 5) (Figure 4). This switch in preference occurred on day 2, with no significant difference in removal rates ( $42 \%$ vs. $58 \%)$. All subcolonies (i.e., replicates) responded similarly to the treatment (GLMM $M_{\text {choice assay, }}$ effect of subcolony: $F_{2,18}=0.00 ; p>0.05$ ).

Leaf-cutting ant performance in shearinine $L$ (1) treated versus control subcolonies was investigated for $10 \mathrm{~d}$, starting on day 2 of the choice assay, by determining the daily amount of waste deposition (fresh weight) and worker mortality (number of dead ants). Subcolonies treated with shearinine $L$ (1) revealed similar worker mortality and waste deposition as the control subcolonies, and both measures of colony performance remained constant throughout the $10 \mathrm{~d}$ experiment

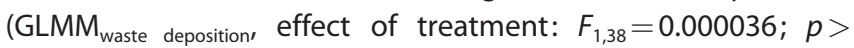
0.05 ; effect of day: $F_{9,38}=1.37 ; p>0.05$; effects of day in combination with treatment: $F_{9,38}=0.62 ; p>0.05$; effect of subcolony: $F_{2,38}=1.99 ; p>0.05 ; G L M M_{\text {worker mortality }}$ effect of treatment: $F_{1,38}=0.34 ; p>0.05$; effect of day: $F_{9,38}=8.42 ; p>0.05$; effects of day in combination with treatment: $F_{9,38}=0.62 ; p>0.05$; effect of subcolony: $F_{2,38}=8.42 ; p<0.001$ ).

\section{Discussion}

How the major pathogen of leaf-cutting ants, E. weberi, overwhelms L. gongylophorus and thus threatens survival of the ants has not been addressed in detail so far. Using metabolite profiling by LC-DAD-MS of E. weberi extracts, we identified seven of the major compounds produced by both $E$. weberi and E. aspergilloides as shearinines (1-5), cycloarthropsone (6), and emodin (7) (Figure 1, S1, and S31 A). The major metabolites $\mathbf{1 - 3}, \mathbf{6}$, and 7 were also detected when L. gongylophorus growing on PDA medium was challenged with E. weberi (Figure S31B). Since we did not observe the formation of other secondary metabolites in these co-cultivation experimentsthat might have been produced in response to the presence of L. gongylophorus, we assumed that the identified com- 
pounds comprise those used by $E$. weberi to overpower the leaf-cutting ants' nest.

The finding of shearinines is in line with Boya et al., who recently observed shearinines $D, F$, and $J$ in a co-cultivation experiment with mutualistic Streptomyces symbionts of Acromyrmex echinatior leaf-cutting ants against E. weberi TZ49. ${ }^{[12]}$ Also, genome mining using antismash ${ }^{[21]}$ revealed that the $E$. weberi genome ${ }^{[8]}$ comprises genes involved in indole terpenoid biosynthesis fitting to the formation of shearinines.

However, our Escovopsis strains mainly released two novel shearinines $L(1)$ and $M(2)$ alongside the known shearinines $D$ (3), E (4) and A (5) with maximal production after 9-12 d of growth on SFM agar plates (Figure 1). Shearinines have been originally identified from Eupenicillium shearii. ${ }^{[13]}$ Shearinines exhibit anticancer activities, ${ }_{i}^{[15]}$ they block large-conductance calcium-activated potassium channels, ${ }^{[14]}$ and are known to act against insects. ${ }^{[13]}$ Furthermore, shearinines have been found to suppress Candida albicans biofilm formation and potentiate the antifungal activity of the polyene macrolide antifungal amphotericin B. ${ }^{[22]}$ However, in agar diffusion assays against $L$. gonglyophorus $0.3 \mu \mathrm{mol}$ shearinine $L$ (1) did not stop the growth of L. gongylophorus.

Due to the reported activity of shearinines against insects, ${ }^{[13]}$ we suspected that they may rather act against the leaf-cutting ants than the garden fungus and thus indirectly weaken the fitness of the garden fungus. In order to address how shearinines may affect the leaf-cutting ants' colony we investigated how $A$. octospinosus subcolonies responded to the major component shearinine $L$ (1). Shearinine $L$ (1) impregnated oat flakes and control oat flakes were offered to $A$. octospinosus. Initially, $A$. octospinosus accepted both treated and untreated flakes even preferring the shearinine $L(1)$ treated flakes. Consequently, the ants did not immediately recognize shearinine $L$ (1) as detrimental. However, after a short learning period the ants clearly rejected shearinine $L$ treated oat flakes as substrate for L. gongylophorus from day 3 onwards (Figure 4). Thus, A. octospinosus ants quickly learned to avoid shearinine $L(1)$ treated oat flakes. However, when we evaluated the state of the subcolonies (daily waste production) as well as the death rate of A. octospinosus workers no clear signs of a direct toxicity of shearinine $L$ (1) could be observed indicating that shearinine $L$ (1) does not quickly kill $A$. octospinosus workers. Still after $2 \mathrm{~d}$ $A$. octospinosus ants strongly rejected the substrate treated with shearinine $L(1)$.

A similar delayed rejection and avoidance learning-most likely induced by $L$. gongylophorus-has been studied previously with antifungal (cycloheximide) treated plant substrate ${ }^{[23]}$ as well as defense-induced plant material. ${ }^{[20]}$

Currently, it remains elusive how $A$. octospinosus workers learn to avoid shearinine $L$ (1) a major compound produced by $E$. weberi. It is conceivable that the avoidance is induced due to a signal by L. gongylophorus indicating a detrimental substrate or a cue that links to the pathogen E. weberi. Alternatively, the ants recognize themselves, for example, during the processing of the oat flakes into small pieces for their garden fungus, that shearinine $L(1)$ is noxious or associated with the detrimental $E$. weberi.
In contrast to shearinine $L$ (1), both cycloarthropsone (6) and emodin (7) strongly inhibited the growth of L. gongylophorus in agar diffusion assays (Figure 3, Figure S35, Figure S36) suggesting that Escovopsis fungi can make use of these polyketides to overpower L. gongylophorus. Cycloarthropsone (6) has been identified from Arthropsis truncata. ${ }^{[16]}$ However, its biological activity has not been evaluated so far. In contrast, emodin (7), that is produced by plants and microorganisms, is well known for its wide range of pharmacological activities, such as antibacterial (against Bacillus subtilis), ${ }^{[24]}$ antifungal (against Alternaria, Fusarium, Fomesannosus etc.), ${ }_{1}^{[25]}$ antiviral, ${ }^{[26]}$ anticancer, ${ }^{[27]}$ anti-inflammatory, ${ }^{[28]}$ and anti-ulcerogenic properties. ${ }^{[29]}$ Thus, emodin (7) may help Escovopsis to outcompete other fungi that are known to occur in the leaf-cutting ants' nests. ${ }^{[30]}$ However, our test strain for alternative pathogens, Fusarium equiseti, was not clearly inhibited in our agar diffusion assays $(0.3 \mu \mathrm{mol}$, Figure S34). But emodin (7) $(0.3 \mu \mathrm{mol})$ strongly inhibited some Actinomyces symbionts of leaf-cutting ants (Figure S32 and S33) indicating its use to fight against the protectors of the fungus garden.

\section{Conclusions}

The production of emodin (7) and cycloarthropsone (6) helps E. weberi to overpower its prey L. gongylophorus as well as to fight against the defenders, in particular the mutualistic Actinomyces symbionts.

However, A. octospinosus leaf-cutting ants-probably communicated by $L$. gongylophorus - can quickly learn to recognize shearinine $L$ (1) from $E$. weberi and thus may initiate defense reactions against the pathogen demonstrating the arms race between the organisms.

With a multitude of organisms - the ants, their garden fungus, the mutualistic microbial symbionts, and pathogens, in particular E. weberi,-comprising the leaf-cutting ants' microcosmos, a detailed picture of the complex interactions can only be obtained if the involved chemicals, signaling compounds, toxins and antimicrobials, of the different partners are identified and their ecological function is addressed. In addition to straightforward in vitro bioassays, (behavioral) studies under close to natural conditions can help to reveal a more complete picture of the interactions and the diverse functions of the involved metabolites-as observed in the case of shearinine $L(1)$.

Future experiments are needed to further reveal the biological function(s) of secondary metabolites from Escovopsis and thus shed more light on the fascinating interactions in the leaf-cutting ant microcosmos.

\section{Experimental Section}

\section{General}

Chemicals were purchased from Sigma Aldrich. IR detection was conducted on an ALPHA FT-IR spectrometer (Bruker, Germany). CD spectra were recorded with a Jasco J-815 CD-spectrometer and the optical rotation was determined with a Jasco P2000 polarimeter. 
NMR spectra were recorded with a Bruker AVANCE AV-600 NMR spectrometer or a Bruker AV-600 NMR spectrometer fitted with a $\mathrm{TCl}$ cryoprobe (Bruker, Karlsruhe, Germany). Chemical shifts $\delta$ (ppm) were referenced to the solvent signals ( ${ }^{1} \mathrm{H} N M R \mathrm{CD}_{3} \mathrm{OD}$ : 3.31, ${ }^{13} \mathrm{C}$ NMR $\mathrm{CD}_{3} \mathrm{OD}$ : 49.00, ${ }^{1} \mathrm{H}$ NMR $\left[\mathrm{D}_{6}\right.$ ] acetone: $2.05,{ }^{13} \mathrm{C}$ NMR $\left[\mathrm{D}_{6}\right.$ ] acetone: $\left.29.84 \mathrm{ppm}\right)$. HR-ESI-MS were acquired using a Bruker Impact II (Bruker, Daltonics, Germany). UV/Vis spectra were recorded using an Implen nanophotometer. For column chromatography silica gel (40-63 mesh, Macherey-Nagel, Germany) and Sephadex LH-20 (GE Healthcare, Uppsala, Sweden) were used. Spots were detected under UV light and visualized by spraying with $5 \% \mathrm{H}_{2} \mathrm{SO}_{4}$ in $\mathrm{EtOH}(\mathrm{v} / \mathrm{v})$, followed by heating the plates. Silica GF254 (Merck $\mathrm{KGaA}$, Germany) was used for analytical thin-layer chromatography. High performance liquid chromatography (HPLC) was performed on an Agilent 1100 liquid chromatography system fitted with a Phenomenex polar-RP column $(250 \times 2 \mathrm{~mm}, 4 \mu \mathrm{m}$ or $250 \times 4.6 \mathrm{~mm}$, $4 \mu \mathrm{m})$ hyphenated to a Gilson FC204 fraction collector. LC-MS measurements were conducted with an Agilent 1100 HPLC connected to a Finnigan MAT LCQ. For GC-MS analysis, a Thermofisher Trace GC Ultra hyphenated with an ISQ was used.

\section{Fungal and microbial cultures}

E. weberi CBS 110660 and E. aspergilloides CBS 423.93 were obtained from the Westerdijk Fungal Biodiversity Institute (Utrecht, the Netherlands). F. equiseti FSU 5459 was from the Jena Microbial Resource Collection (JMRC, Jena, Germany). P. fastigiata DSM 2692 originated from the German Collection of Microorganisms and Cell Cultures (Braunschweig, Germany). The Streptomyces and Pseudonocardia strains used for bioassays were isolates from $A$. volcanus, A. octospinosus, and A. echinatior leaf-cutting ants. ${ }^{[6, h]}$ L.gongylophorus was isolated from an A. octospinosus nest maintained at University of Kaiserslautern by Dr. Rainer Wirth and verified by $18 \mathrm{~S}$ rDNA sequencing (Supporting Information). L. gongylophorus was grown on potato dextrose agar medium (PDA) purchased from Sigma Aldrich at $30^{\circ} \mathrm{C}$. All other strains were cultivated on soy flour medium (SFM) agar plates $(20 \mathrm{~g}$ soy flour, $20 \mathrm{~g}$

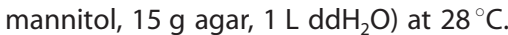

\section{A. octospinosus leaf-cutting ants}

The A. octospinosus ants originated from Paratebueno, Medina, Colombia $\left(4^{\circ} 23^{\prime} 38.4^{\prime \prime} \mathrm{N} 73^{\circ} 16^{\prime} 07.5^{\prime \prime} \mathrm{W}\right)$. The ants were kept as laboratory culture at the Technical University Kaiserslautern at $25^{\circ} \mathrm{C}$ under a $12 \mathrm{~h}: 12 \mathrm{~h}$ light:dark regime. The ants were offered fresh blackberry leaves as substrate for $L$. gongylophorus.

\section{Extraction and isolation of secondary metabolites from $E$. weberi}

Secondary metabolites were isolated from $E$. weberi grown on SFM agar plates at $28^{\circ} \mathrm{C}$ for $14 \mathrm{~d}$. 20 SFM agar plates $(9 \mathrm{~cm})$ were cut in pieces and extracted with ethyl acetate $(500 \mathrm{~mL})$ for $2 \mathrm{~h}$ twice. After filtration, the combined solvent extracts were dried in vacuo. The crude extract $(0.459 \mathrm{~g})$ was subjected to silica gel column chromatography (column dimension $3 \times 25 \mathrm{~cm}$ ) eluting with $n$-hexane and ethyl acetate $(7: 1,5: 1,3: 1,2: 1,1: 1,1: 3,1: 6,0: 1 \mathrm{v} / \mathrm{v}, 200 \mathrm{~mL}$ each) to give six fractions (Fr1-Fr6). Fr2 (11.3 mg) was passed three times through a Sephadex $\mathrm{LH}-20$ column with $90 \% \mathrm{MeOH}$ to give pure compound 7 (3.1 mg). Likewise, Fr3 $(16 \mathrm{mg})$ was subjected to Sephadex LH-20 column chromatography with $90 \% \mathrm{MeOH}$ and further purified by HPLC using a Phenomenex polar RP column ( $250 \times$ $4.6 \mathrm{~mm}, 4 \mu \mathrm{m})$ at a flow rate of $0.8 \mathrm{~mL} \mathrm{~min}^{-1}$ eluting the compounds with a gradient of solvent $\mathrm{A}\left(\mathrm{H}_{2} \mathrm{O} 0.1 \%\right.$ acetic acid) and solvent $\mathrm{B}$ (MeOH $0.1 \%$ acetic acid). HPLC conditions: 2 min 15\% B, in $28 \mathrm{~min}$ to $100 \% \mathrm{~B}, 5 \mathrm{~min} 100 \% \mathrm{~B}$. Compound $1(1.90 \mathrm{mg})$ and compound $3(1.45 \mathrm{mg})$ were obtained. A similar fractionation procedure was used for Fr4 $(31.0 \mathrm{mg})$ to yield compounds 4 (1.3 mg), $5(1.25 \mathrm{mg})$ and $6(3.2 \mathrm{mg})$. Furthermore, $\operatorname{Fr} 5(6.5 \mathrm{mg})$ was directly purified by HPLC with a gradient of solvent $\mathrm{A}\left(\mathrm{H}_{2} \mathrm{O} 0.1 \%\right.$ acetic acid) and solvent $\mathrm{B}$ (MeOH $0.1 \%$ acetic acid): HPLC conditions: ( 2 min $50 \%$ B, in 28 min to $100 \%$ B, 5 min $100 \%$ B) to yield compound 2.

\section{Spectroscopic data of shearinine $L$ (1)}

$1.9 \mathrm{mg}$, white amorphous powder; IR $(\mathrm{KBr}): \tilde{v}_{\max }=3441,2971,2617$, 2343, 2051, 1668, 1462, 1365, 1253, $1149 \mathrm{~cm}^{-1}$; UV $\left(\mathrm{CH}_{3} \mathrm{OH}\right) \lambda_{\max }=$ $255,329 \mathrm{~nm} ;{ }^{1} \mathrm{H}$ NMR $\left(600 \mathrm{MHz}, \mathrm{CD}_{3} \mathrm{OD}\right) \delta_{\mathrm{H}}=7.35(1 \mathrm{H}, \mathrm{s}, \mathrm{H}-30)$, $7.16(1 \mathrm{H}, \mathrm{s}, \mathrm{H}-20), 5.95(1 \mathrm{H}, \mathrm{d}, J=2.96, \mathrm{H}-27), 5.93(1 \mathrm{H}, \mathrm{brs}, \mathrm{H}-11)$, $5.72(1 \mathrm{H}, \mathrm{dt}, J=6.3, J=2.2, \mathrm{H}-6), 4.12(1 \mathrm{H}, \mathrm{s}, \mathrm{H}-9), 3.14(1 \mathrm{H}, \mathrm{d}, J=$ 18.5, H-5), $3.09(1 \mathrm{H}, \mathrm{dd}, J=15.3, J=9.2, \mathrm{H}-22), 2.86-2.91(1 \mathrm{H}, \mathrm{m}, \mathrm{H}-$ 23), $2.67(1 \mathrm{H}, \mathrm{dd}, J=12.5, J=6.2, \mathrm{H}-17), 2.60-2.64(1 \mathrm{H}, \mathrm{m}, \mathrm{H}-22)$, 2.52-2.59 (1 H, m, H-16), 2.30-2.35 (1 H, m, H-17), 2.27-2.31 (m, H5), 1.96-2.02 $(1 \mathrm{H}, \mathrm{m}, \mathrm{H}-15), 1.89(1 \mathrm{H}, \mathrm{d}, J=13.6, \mathrm{H}-14), 1.66-1.73$ $(1 \mathrm{H}, \mathrm{m}, \mathrm{H}-14), 1.61(1 \mathrm{H}, \mathrm{d}, J=12.2, \mathrm{H}-15), 1.38(3 \mathrm{H}, \mathrm{s}, \mathrm{H}-40), 1.30-$ $1.36(12 \mathrm{H}, \mathrm{m}, \mathrm{H}-32, \mathrm{H}-36, \mathrm{H}-37, \mathrm{H}-39), 1.27(3 \mathrm{H}, \mathrm{s}, \mathrm{H}-35), 1.13(3 \mathrm{H}$ s, H38), 0.94 ppm ( $3 \mathrm{H}$, brs, H-33); ${ }^{13} \mathrm{C}$ NMR $\left(150 \mathrm{MHz}, \mathrm{CD}_{3} \mathrm{OD}\right) \delta_{\mathrm{C}}=$ 197.6 (C-10), 156.8 (C-7), 155.1 (C-2), 146.5 (C-12), 141.9 (C-31), 141.5 (C-29), 137.0 (C-28), 133.7 (C-21), 128.1 (C-19), 119.4 (C-27), 117.1 (C-11), 117.0 (C-18), 114.3 (C-20), 113.3 (C-6), 104.1 (C-30), 87.1 (C-9), 76.3 (C-24), 76.1 (C-13), 74.6 (C-34), 74.4 (C-26), 52.1 (C-3), 50.7 (C-16), 50.3 (C-23), 44.3 (C-4), 34.0 (C-22), 33.4 (C-14), 32.4 (C5), 32.2 (C-39), 30.6 (C-40) 30.2 (C37), 28.0 (C-17), 26.8 (C-36), 26.7 (C-35), 22.7 (C-15), 22.3 (C-38), 21.3 (C-33), 16.6 ppm (C-32); HR-ESIMS: calcd for $\mathrm{C}_{37} \mathrm{H}_{44} \mathrm{NO}_{5}$ : $582.32250[\mathrm{M}-\mathrm{H}]^{-}$; found: 582.32053 .

\section{Spectroscopic data of shearinine M (2)}

$0.98 \mathrm{mg}$, white amorphous powder; IR $(\mathrm{KBr}): \tilde{v}_{\max }=3409,3297$, $2987,2369,2183,1668,1476,1386,1309,1186 \mathrm{~cm}^{-1}$; UV $\left(\mathrm{CH}_{3} \mathrm{OH}\right)$ $\lambda_{\max }=259,328 \mathrm{~nm} ;{ }^{1} \mathrm{H}$ NMR $\left(600 \mathrm{MHz}, \mathrm{CD}_{3} \mathrm{OD}\right) \delta_{\mathrm{H}}=7.37(1 \mathrm{H}, \mathrm{s}, \mathrm{H}-$ 30), $7.36(1 \mathrm{H}, \mathrm{s}, \mathrm{H}-20), 5.99(1 \mathrm{H}, \mathrm{d}, J=3.0, \mathrm{H}-27), 5.94(1 \mathrm{H}, \mathrm{brs}, \mathrm{H}-$ 11), 5.72-5.75 $(1 \mathrm{H}, \mathrm{m}, \mathrm{H}-6), 4.90(1 \mathrm{H}, \mathrm{brd}, J=5.7, \mathrm{H}-22), 4.12(1 \mathrm{H}, \mathrm{s}$, $\mathrm{H}-9), 3.16(1 \mathrm{H}, \mathrm{d}, J=18.4, \mathrm{H}-5), 2.69(1 \mathrm{H}, \mathrm{dd}, J=12.5, J=6.2, \mathrm{H}-17)$, $2.67(1 \mathrm{H}, \mathrm{dd}, J=5.7, J=2.8, \mathrm{H}-23), 2.65-2.67(1 \mathrm{H}, \mathrm{m}, \mathrm{H}-16), 2.36-$ $2.41(1 \mathrm{H}, \mathrm{m}, \mathrm{H}-17), 2.30-2.36(1 \mathrm{H}, \mathrm{m}, \mathrm{H}-5), 1.99-2.06(1 \mathrm{H}, \mathrm{m}, \mathrm{H}-15)$, 1.90-1.96 $(1 \mathrm{H}, \mathrm{m}, \mathrm{H}-14), 1.76-1.83(1 \mathrm{H}, \mathrm{m}, \mathrm{H}-14), 1.67(1 \mathrm{H}, \mathrm{d}, J=$ $12.3, \mathrm{H}-15), 1.46(3 \mathrm{H}, \mathrm{s}, \mathrm{H}-37), 1.37(3 \mathrm{H}, \mathrm{s}, \mathrm{H}-40), 1.34(3 \mathrm{H}, \mathrm{s}, \mathrm{H} 32)$, 1.32-1.34 (6 H, s, H-36, H-39), $1.27(3 \mathrm{H}, \mathrm{s}, \mathrm{H}-35), 1.15(3 \mathrm{H}, \mathrm{s}, \mathrm{H} 38)$, $0.99 \mathrm{ppm}(3 \mathrm{H}, \mathrm{s}, \mathrm{H}-33) ;{ }^{13} \mathrm{C}$ NMR $\left(150 \mathrm{MHz}, \mathrm{CD}_{3} \mathrm{OD}\right) \delta_{\mathrm{C}}=197.6(\mathrm{C}-$ 10), 156.7 (C-7), 155.5 (C-2), 146.5 (C-12), 142.9 (C-31), 139.2 (C-29), 137.3 (C-28), 132.0 (C-21), 128.2 (C-19), 120.2 (C-27), 117.4 (C-18), 117.1 (C-11), 114.2 (C-20), 113.3 (C-6), 103.6 (C-30), 87.2 (C-9), 76.7 (C-22), 76.0 (C-13), 75.6 (C-24), 74.7 (C-34), 74.1 (C-26), 60.3 (C-23), 52.2 (C-3), 50.7 (C-16), 44.3 (C-4), 33.4 (C-14), 32.3 (C-5), 32.2 (C-39), 30.4 (C-40), 30.3 (C-37), 28.0 (C-17), 26.8 (C-36), 26.7 (C-35), 23.7 (C38), 22.4 (C-15), 21.2 (C-33), 16.6 ppm (C-32); HR-ESI-MS: calcd for $\mathrm{C}_{37} \mathrm{H}_{44} \mathrm{NO}_{6}: 598.31741[\mathrm{M}-\mathrm{H}]^{-}$; found: 598.31763.

\section{Agar diffusion assays}

L. gongylophorus, F. equiseti, P. fastigiata, Streptomyces sp. 25_4, Streptomyces sp. 28_1, Streptomyces sp. 26_3 and Pseudonocardia $s p$. Ao19 were used as test organisms in the agar diffusion assays against the purified secondary metabolites or reference compounds. For the agar diffusion assays, $100 \mu \mathrm{L}$ of mycelium suspensions of the test organisms were spread onto SFM or PDA agar 
plates $(5.5 \mathrm{~cm}$ diameter). Holes ( $6 \mathrm{~mm}$ diameter) were cut into the agar plates into which $50 \mu \mathrm{L}$ of the test solutions or solvent controls $(\mathrm{MeOH})$ were applied. For paper disk diffusion assays, sterilized filter paper disks ( $6 \mathrm{~mm}$ diameter) were impregnated with the test compounds $(0.3,0.66$, and $0.82 \mu \mathrm{mol})$. The test organisms were cultivated at $28^{\circ} \mathrm{C}$ except $L$. gongylophorus, which was grown at $30^{\circ} \mathrm{C}$. The inhibition zones were monitored daily from day 3 to 18. All assays were performed at least in triplicate and were compared to equally prepared solvent controls.

\section{Artificial infection of L. gongylophorus with E. weberi}

L. gongylophorus was cultivated for $15 \mathrm{~d}$ on PDA agar at $30^{\circ} \mathrm{C}$. Then $E$. weberi was added to the plates and both strains were cultivated for additional $7 \mathrm{~d}$. Single plates (three replicates) were extracted with $\mathrm{MeOH}(20 \mathrm{~mL})$, concentrated to $1 \mathrm{~mL}$ and subjected to LC-MS analysis (10 $\mu \mathrm{L}$ injection volume, see above).

\section{Establishment of $A$. octospinosus subcolonies}

Bioassays with $A$. octospinosus leaf-cutting ants were performed with 6 subcolonies ( 3 treatments, 3 controls). Both treatment and control subcolonies were cultivated equally apart from the shearinine $L$ treatment. The subcolonies were established by isolating ca. $400 \mathrm{~cm}^{3}$ of fresh fungus garden from three large laboratory mother colonies. The subcolonies were kept in experimental nests, consisting of 3 plastic boxes (feeding box, fungus garden box, and refuse chamber) connected by plastic tubes. The A. octospinosus subcolonies were kept at $25^{\circ} \mathrm{C}$ under a $12 \mathrm{~h}: 12 \mathrm{~h}$ light:dark regime. All subcolonies were established at least $5 \mathrm{~d}$ before the dual choice experiment and supplied with fresh blackberry leaves.

\section{Coating of oat flakes with shearinine $L$ (1)}

Oat flakes (Blüten-zarte Flocken, Kölln, Germany) of approximately equal size were used for the experiment. The flakes were impregnated with $10 \mu \mathrm{L}$ of shearinine $\mathrm{L}(1)\left(1 \mathrm{mg} \mathrm{mL}^{-1}\right)$ dissolved in acetone and air-dried. Control flakes were prepared analogously applying $10 \mu \mathrm{L}$ acetone.

\section{Dual-choice bioassays ${ }^{[20]}$ with $A$. octospinosus leaf-cutting ants}

For the dual-choice preference tests, 10 treated and 10 control flakes were simultaneously placed $3 \mathrm{~cm}$ apart in the foraging chamber of $A$. octospinosus subcolonies. Control subcolonies were only offered control flakes.

The subcolonies were monitored continuously and the number of flakes carried inside the nest was noted until 8 out of 10 control or shearinine L-treated flakes were picked up or $5 \mathrm{~h}$ had elapsed. All collected flakes were readily processed and incorporated into the fungus garden. The foraging preferences of each subcolony were monitored daily over $5 \mathrm{~d}$. Starting from the second day of feeding preference tests, the number of dead ants (i.e., worker mortality) and the amount of waste deposited by the ants into the waste chamber were recorded daily for $10 \mathrm{~d}$. The influence of shearinine $L(1)$ on the choice behavior of ants, their mortality, and waste deposition was statistically analyzed using general linear mixed models $\left(G L M M_{\text {choice assay }}\right.$ GLMM worker mortality $_{\text {and }}$ aLMM $M_{\text {waste deposition, }}$ respectively) with treatment, day and their interaction as fixed factors and subcolony as random factor. The $F$ values represent the actual test results of the GLMM (equivalent to $F$ values in an Anova); $p$ values are calculated based on these $F$ values. The subscript values are the degrees of freedom. Ant mortality was square- root transformed to achieve homogeneity of variance. Statistical tests were performed with Statistica software (Statistica 13, TIBCO Software Inc., Palo Alto, USA).

\section{Acknowledgements}

We thank Prof. Peter Spiteller and Dr. Thomas Dülcks for HRESI-MS measurements, Natalie Kunz for advice in statistical analysis and Michael Schremel for rearing the ants. We gratefully acknowledge language editing and helpful comments by Dr. Anthony Farlow. We thank Christian Ziegler for the A. octospinosus photo. We are greatly indebted to the Konstanz Research School Chemical Biology funded by the Deutsche Forschungsgemeinschaft for financial support and a doctoral fellowship to B.D.

\section{Conflict of interest}

The authors declare no conflict of interest.

Keywords: antibiotics $\cdot$ cycloarthropsone $\cdot$ emodin $\cdot$ natural products $\cdot$ shearinine

[1] a) T. R. Schultz, S. G. Brady, Proc. Natl. Acad. Sci. USA 2008, 105, 54355440; b) B. Hölldobler, E. O. Wilson, The Ants, Springer, Berlin, 1990.

[2] A. Rodrigues, M. Bacci Jr., U. Mueller, A. Ortiz, F. C. Pagnocca, Microb. Ecol. 2008, 56, 604-614.

[3] F. C. Pagnocca, V. E. Masiulionis, A. Rodrigues, Psyche 2012, 905109.

[4] a) H. Schildknecht, K. Koob, Angew. Chem. Int. Ed. Engl. 1970, 9, 173; Angew. Chem. 1970, 82, 181; b) A. N. M. Bot, D. Ortius-Lechner, K. Finster, R. Maile, J. J. Boomsma, Insectes Soc. 2002, 49, 363-370.

[5] M. Bass, J. Cherrett, Ecol Entomol. 1994, 19, 215-220.

[6] a) C. R. Currie, B. Wong, A. E. Stuart, T. R. Schultz, S. A. Rehner, U. G. Mueller, G.-H. Sung, J. W. Spatafora, N. A. Straus, Science 2003, 299, $386-$ 388; b) A. V. Santos, R. J. Dillon, V. M. Dillon, S. E. Reynolds, R. I. Samuels, FEMS Microbiol. Lett. 2004, 239, 319-323; c) C. Kost, T. Lakatos, I. Boettcher, W.-R. Arendholz, M. Redenbach, R. Wirth, Naturwissenschaften 2007, 94, 821-828; d) A. Rodrigues, R. N. Cable, U. G. Mueller, M. Bacci, F. C. Pagnocca, Antonie van Leeuwenhoek 2009, 96, 331-342; e) S Haeder, R. Wirth, H. Herz, D. Spiteller, Proc. Natl. Acad. Sci. USA 2009, 106, 4742-4746; f) D.-C. Oh, M. Poulsen, C. R. Currie, J. Clardy, Nat. Chem. Biol. 2009, 5, 391 -393; g) J. Barke, R. F. Seipke, S. Gruschow, D. Heavens, N. Drou, M. J. Bibb, R. J. M. Goss, D. W. Yu, M. I. Hutchings, BMC Biol. 2010, 8, 109; h) I. Schoenian, M. Spiteller, M. Ghaste, R. Wirth H. Herz, D. Spiteller, Proc. Natl. Acad. Sci. USA 2011, 108, 1955-1960; i) E. Freinkman, D.-C. Oh, J. J. Scott, C. R. Currie, J. Clardy, Tetrahedron Lett. 2009, 50, 6834-6837; j) C. S. Sit, A. C. Ruzzini, E. B. Van Arnam, T. R. Ramadhar, C. R. Currie, J. Clardy, Proc. Natl. Acad. Sci. USA 2015, 112, $13150-13154$; k) E. B. Van Arnam, A. C. Ruzzini, C. S. Sit, H. Horn, A. A. Pinto-Tomas, C. R. Currie, J. Clardy, Proc. Natl. Acad. Sci. USA 2016, 113, $12940-12945$.

[7] a) S. C. Carreiro, F. C. Pagnocca, O. C. Bueno, M. B. Júnior, M. J. A. Hebling, O.A. da Silva, Antonie van Leeuwenhoek 1997, 71, 243-248; b) C. R. Currie, U. G. Mueller, D. Malloch, Proc. Natl. Acad. Sci. USA 1999, 96, 7998-8002; c) G. Suen, J. J. Scott, F. O. Aylward, S. M. Adams, S. G. Tringe, A. A. Pinto-Tomás, C. E. Foster, M. Pauly, P. J. Weimer, K. W. Barry, PLoS Genet. 2010, 6, e1001129.

[8] T. J. de Man, J. E. Stajich, C. P. Kubicek, C. Teiling, K. Chenthamara, L. Atanasova, I. S. Druzhinina, N. Levenkova, S. S. Birnbaum, S. M. Barribeau, Proc. Natl. Acad. Sci. USA 2016, 113, 3567-3572.

[9] a) N. M. Gerardo, S. R. Jacobs, C. R. Currie, U. G. Mueller, PLoS Biol. 2006, 4, e235; b) N. M. Gerardo, U. G. Mueller, S. L. Price, C. R. Currie, Proc. R. Soc. London Ser. B 2004, 271, $1791-1798$.

[10] H. T. Reynolds, C. R. Currie, Mycologia 2004, 96, 955-959. 
[11] S. S. Varanda-Haifig, T. R. Albarici, P. H. Nunes, I. Haifig, P. C. Vieira, A. Rodrigues, Antonie van Leeuwenhoek 2017, 110, 593-605.

[12] C. A. P. Boya, H. Fernández-Marín, L. C. Mejía, C. Spadafora, P. C. Dorrestein, M. Gutiérrez, Sci. Rep. 2017, 7, 5604.

[13] G. N. Belofsky, J. B. Gloer, D. T. Wicklow, P. F. Dowd, Tetrahedron 1995, 51, 3959-3968.

[14] M. Xu, G. Gessner, I. Groth, C. Lange, A. Christner, T. Bruhn, Z. Deng, X. Li, S. H. Heinemann, S. Grabley, Tetrahedron 2007, 63, 435-444.

[15] O. F. Smetanina, A. I. Kalinovsky, Y. V. Khudyakova, M. V. Pivkin, P. S. Dmitrenok, S. N. Fedorov, H. Ji, J.-Y. Kwak, T. A. Kuznetsova, J. Nat. Prod. 2007, 70, 906-909.

[16] W. A. Ayer, P. A. Craw, J. Neary, Can. J. Chem. 1992, 70, 1338-1347.

[17] K. A. Seifert, R. A. Samson, I. H. Chapela, Mycologia 1995, 87, 407-413.

[18] L. A. Meirelles, Q. V. Montoya, S. E. Solomon, A. Rodrigues, PLoS One 2015, 10, e0112067.

[19] A. E. F. Little, C. R. Currie, Biol. Lett. 2007, 3, $501-504$.

[20] T. Thiele, C. Kost, F. Roces, R. Wirth, J. Chem. Ecol. 2014, 40, 617-620.

[21] M. H. Medema, K. Blin, P. Cimermancic, V. de Jager, P. Zakrzewski, M. A. Fischbach, T. Weber, E. Takano, R. Breitling, Nucleic Acids Res. 2011, 39, W339-W346.

[22] J. You, L. Du, J. B. King, B. E. Hall, R. H. Cichewicz, ACS Chem. Biol. 2013, $8,840-848$.
[23] a) P. Ridley, P. E. Howse, C. W. Jackson, Experientia 1996, 52, 631-635; b) H. Herz, B. Hölldobler, F. Roces, Behav. Ecol. 2008, 19, 575-582.

[24] H. Levin, R. Hazenfratz, J. Friedman, D. Palevitch, M. Perl, Phytother. Res. 1988, 2, 67-69.

[25] I. Izhaki, New Phytol. 2002, 155, 205 - 217.

[26] D. L. Barnard, J. H. Huffman, J. L. Morris, S. G. Wood, B. G. Hughes, R. W. Sidwell, Antiviral Res. 1992, 17, 63-77.

[27] A. Liu, H. Chen, W. Wei, S. Ye, W. Liao, J. Gong, Z. Jiang, L. Wang, S. Lin, Oncol. Rep. 2011, 26, $81-89$.

[28] M.-Y. Park, H.-J. Kwon, M.-K. Sung, Biosci. Biotechnol. Biochem. 2009, 73, $828-832$.

[29] R. K. Goel, G. Das Gupta, S. N. Ram, V. B. Pandey, Indian J. Exp. Biol. 1991, 29, 230-232.

[30] A. Rodrigues, F. C. Pagnocca, M. Bacci, M. J. A. Hebling, O. C. Bueno, L. H. Pfenning, Folia Microbiol. 2005, 50, $421-425$. 